\title{
Observing ULXs with VLBI: the quest for intermediate-mass black holes
}

\section{Zsolt Paragi*}

Joint Institute for VLBI in Europe

E-mail: zparagiejive.nI

\section{Michael A. Garrett}

Joint Institute for VLBI in Europe

E-mail: garrettejive.nl

\section{Andrew D. Biggs}

UK Astronomy Technology Centre

E-mail: adberoe.ac.uk

\begin{abstract}
Some of the most extreme ultra-luminous X-ray sources (ULX) are good candidates for the long-sought missing link between stellar-mass and supermassive black holes. These hypothetic intermediate-mass black holes (IMBH) may be revealed by very high angular resolution very long baseline interferometry (VLBI) imaging. We show that these objects are naturally compact and bright enough to be detected with present-day technology. We estimate the minimum mass of black holes in nearby galaxies detectable by the European VLBI Network (EVN), and calculate the expected radio flux densities for various X-ray luminosities. Using recent EVN observations of M82 X-1, we derive an upper limit to the black hole mass of $\sim 500 \mathrm{M}_{\odot}$. We conclude that a relativistic jet cannot be responsible for beaming the X rays in this ULX.
\end{abstract}

VI Microquasar Workshop: Microquasars and Beyond

September 18-22 2006

Società del Casino, Como, Italy

\footnotetext{
* Speaker.
} 


\section{Radio observations of ULXs}

There have been some attempts to study ultra-luminous X-ray sources in the radio regime with connected-element interferometers. ULXs are very powerful and highly variable objects in nearby galaxies, the majority being unrelated to the central nuclear source. Still, the observed luminosities greatly exceed the Eddington limit for a stellar-mass object [1,2]. From the variability and the observed quasi-periodic oscillations (in the case of M82 X-1 [3] ) it is clear that they are compact accreting objects. A stellar-mass microquasar would be undetectable in the radio [四, unless in a strong flaring state. However, if some of these sources are powered by intermediate-mass black holes of $\sim 1000 \mathrm{M}_{\odot}$, as their X-ray luminosities indicate [田], one may expect to see associated powerful radio sources.

The first systematic radio study of ULXs was carried out with the VLA at $5 \mathrm{GHz}$ by Körding et al. [5]. They monitored the nine closest sources at eight epochs, and found for most no flaring or continuous radio emission. Two radio detections in M82 were likely supernova remnants (SNR) and unrelated to the ULXs, but that could not be confirmed from the VLA data alone. There were other programmes targeting individual sources. Some of the detected radio counterparts were shown to be resolved, as in the case of Holmberg II [6]. Very recent observations with the Australian Compact Array (ATCA) resulted in discoveries of two new compact radio near ULX positions [7, 8]. These were unresolved with ATCA and had a steep spectral index, indicating that they are either supernova remnants, or related to a radio jet-lobe system. Obviously, higher resolution observations are desirable to unveil the nature of these objects.

\section{Yes, but can we detect IMBHs with VLBI?}

About half-a-dozen sources -the most extreme ones- show a low-energy excess in X rays that may be due to thermal radiation from an accretion disk [9]. The estimated disk temperatures are much lower than expected for stellar-mass systems, indicating that they may represent the IMBH sub-class of the ULX population. While this interpretation is debated by some authors, it seems reasonable to assume that the most powerful and variable ULXs are the most massive ones.

The ultimate evidence for an accreting IMBH may be the detection of synchrotron self-absorbed radio emission from relativistic compact jets associated with these systems. In addition, radio observations provide a new way of constraining the black hole mass. To estimate the expected flux densities one may use the empirical formula of the fundamental black hole activity plane, which relates the X-ray and radio luminosities to the black hole mass [10]. For the most luminous sources with $L_{\mathrm{X}} \sim 3 \times 10^{40} \mathrm{erg} \mathrm{s}^{-1}$ in nearby galaxies like M82 ( $\mathrm{D}=3-4 \mathrm{Mpc}$ ), a black hole of 300-1000 $\mathrm{M}_{\odot}$ should have a radio flux density of $\sim 50-150 \mu \mathrm{Jy}$ at $\mathrm{cm}$ wavelengths. The angular size of such a faint synchrotron self-absorbed radio jet with a brightness temperature of $T_{\mathrm{B}} \sim 10^{12} \mathrm{~K}$ is at the microarcsecond level, and would be unresolved in VLBI observations.

Reaching the 50-150 $\mu \mathrm{Jy}$ regime with the European VLBI Network (EVN, [1]]) may be achieved routinely at data rates of $1 \mathrm{Gbps}$, provided there are good calibrators nearby for high quality phase-reference imaging. We calculated the minimum black hole mass that is still detectable in nearby galaxies, for various integration times (Fig. 1.). One can see that an IMBH with several hundred solar masses could not remain undetected in the Galactic neighbourhood. The expected 


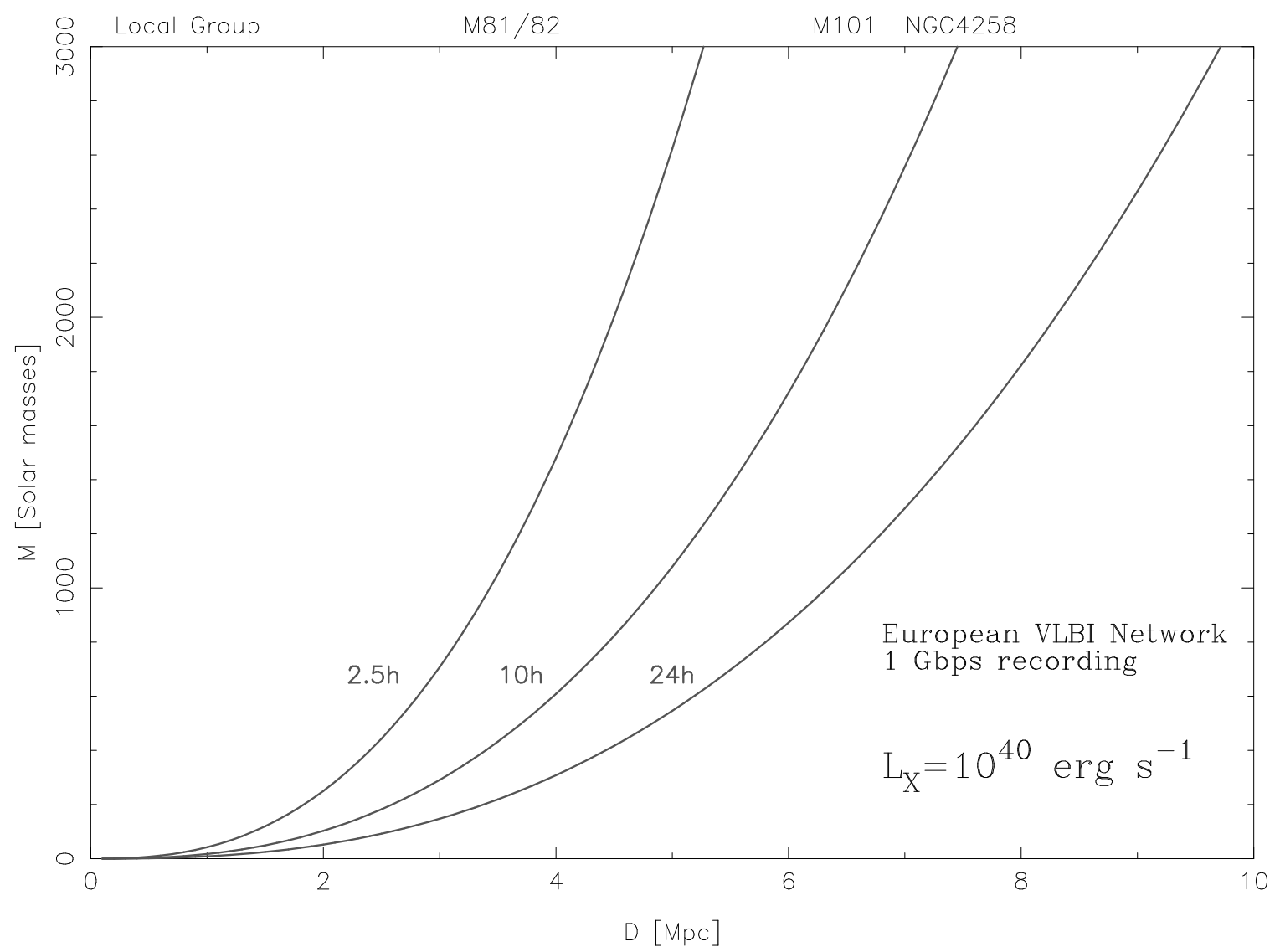

Figure 1: The minimum detectable black hole mass with the EVN in nearby galaxies. The calculations were done at $5 \mathrm{GHz}$ for sources with an X-ray luminosity of $L_{\mathrm{X}}=10^{40} \mathrm{erg} \mathrm{s}^{-1}$, using the formula by T. Maccarone [12]. The $5 \sigma$ detection limits were calculated for various on-source integration times assuming $1 \mathrm{Gbps}$ recording.

radio flux for systems with various X-ray luminosities is given in Fig. 2., assuming a black hole mass of $1000 \mathrm{M}_{\odot}$. Some of the most extreme ULXs are also indicated. It can be seen that M82 $\mathrm{X}-1$ is the best target for VLBI.

\section{Observations of M82 X-1 with the EVN}

We observed M82 with the EVN at $1.6 \mathrm{GHz}$ on 2005 October 27, with an array consisting of Cambridge, Effelsberg, 76m Lovell Telescope (Jodrell Bank), Medicina, Noto, Onsala, 70m Robledo and Westerbork. The total observing time was 15 hours at a sustained data rate of $1 \mathrm{Gbps}$. The correlation took place in JIVE. We used 128 spectral channels and 1s integration time for minimising time and bandwidth smearing effects on the map. In this way we increased the field of view of our observations and were able to image all the compact sources in the central part of M82.

So far only five compact objects have been detected with VLBI in M82 [13. We imaged these, and identified three additional supernova remnant (SNR) candidates in our maps. One of these is located at RA=9h 55m 50.0487s, DEC $=+69$ 40' 45.942" (J2000), well within the 2.1 arcsecond 3sigma error box of M82 X-1 [14]. This position is in agreement with the likely supernova remnant 


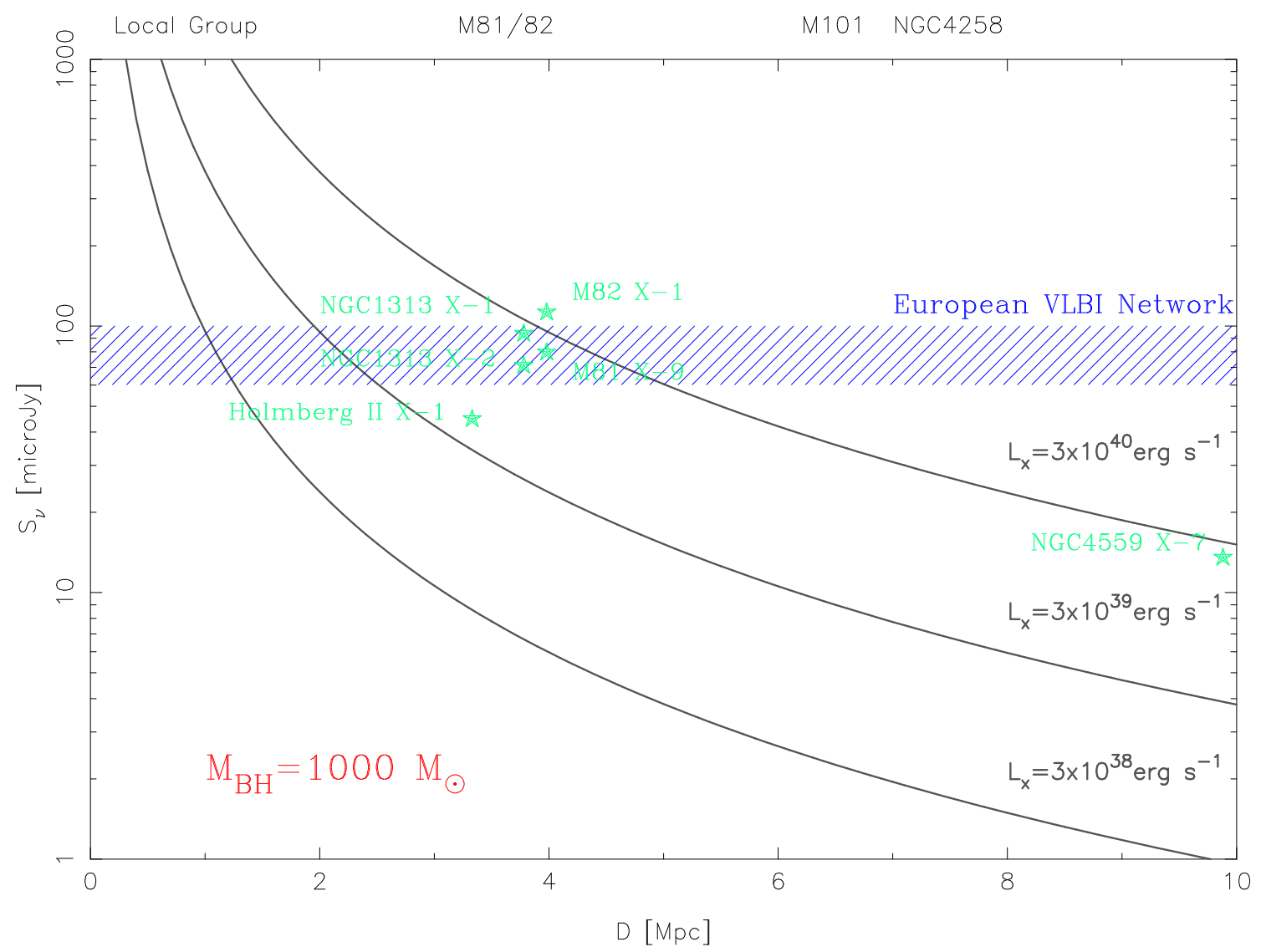

Figure 2: The estimated radio flux of $1000 \mathrm{M}_{\odot}$ IMBHs, for various X-ray luminosities. Some of the most extreme ULXs are also indicated. The striped area shows the regime where these radio sources may be still easily detected in a normal EVN run at 1 Gbps recording.

M82 41.29+59.7 [15]. The radio structure of 41.29+59.7 is resolved, and although it resembles a jet-lobe morphology, it is too bright and well resolved, in contrast with the very small angular size expected for a compact self-absorbed jet. Recent deep Multi-Element Radio-Linked Interferometer Network (MERLIN) observations seem to confirm its SNR nature (T. Muxlow, priv. comm.).

We carefully searched the 2.1 arcsecond CHANDRA error box of M82 X-1 for additional, lower signal-to-noise ratio radio sources. No such sources were found with a $4.5 \sigma$ upper limit of $67 \mu \mathrm{Jy} / \mathrm{beam}$. In particular, we looked for a recently discovered likely new variable source reported by Kaaret et al. [17]. At the position of this faint VLA source we detect no radio emission with a $3.5 \sigma$ upper limit of $50 \mu \mathrm{Jy} / \mathrm{beam}$. At centimeter wavelengths, for the typical X-ray luminosity of $L_{\mathrm{X}}=3 \times 10^{40} \mathrm{erg} \mathrm{s}^{-1}$ and assuming D=3.9 kpc distance for M82 [17], these place an upper limit of about $400-600 \mathrm{M}_{\odot}$ on the black hole mass of M82 X-1. We note that this number is an indicative value rather than a rigorous upper limit.

\section{Are there IMBHs?}

There are a number of difficulties with the IMBH hypothesis. In particular, the formation of IMBHs in sufficient numbers is problematic, leading to the conclusion that the majority of ULXs 


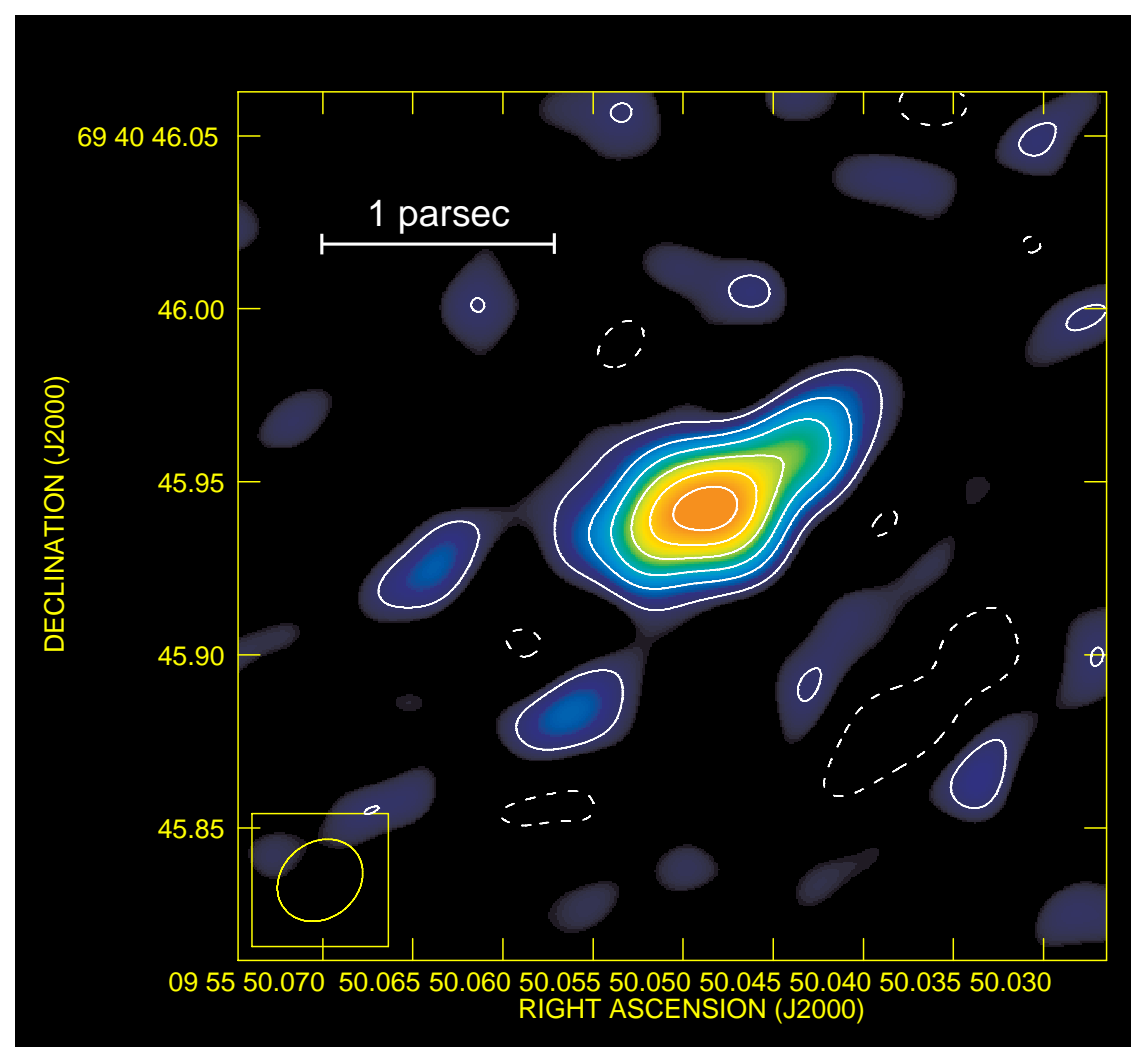

Figure 3: EVN image of M82 41.29+59.7, near the position of M82 X-1 at $1.6 \mathrm{GHz}$. The source shows an elongated structure, resembling a radio jet-lobe. However, deep MERLIN observations show an SNR morphology, and the radio source is unlikely to be related to the ULX. The peak brightness is $281 \mu \mathrm{Jy} / \mathrm{beam}$. The contour levels are $-0.3,0.3,0.65,1.0,1.5,2.0$, and 2.5 times $100 \mu \mathrm{Jy} / \mathrm{beam}$. The restoring beam is $26 \times 21$ milliarcsecond with a position angle of $P A=-51$ degrees. The off-source rms noise is $15 \mu \mathrm{Jy} / \mathrm{beam}$.

are in fact stellar-mass $\mathrm{X}$-ray binaries, where the $\mathrm{X}$ rays from the accretion disk are geometrically beamed [18]. Other suggestions are that these sources accrete at highly super-Eddington rates [19, 20,21], or that the $X$ rays are coming from a relativistically beamed jet [22,23]. A Galactic example of a highly super-Eddington source with likely beaming of X rays may be SS433 [24,25]. The reason why it is not an ULX itself is that we look at the source at a very large angle from the axis.

In this paper we show that the most extreme ULX, M82 X-1 is unlikely to be related to a very massive $\left(\mathrm{M} \gg 500 \mathrm{M}_{\odot}\right)$ intermediate-mass black hole. Future observations may provide stronger limits. As we go deeper and deeper in sensitivity, we must detect these systems, if they exist. We note that VLBI is particularly useful for this task, because it has the power to resolve extended sources, and thus differentiate between HII regions, SNRs, and compact jets. 


\section{References}

[1] E. J. M. Colbert, R. F. Mushotzky, Astrophys. J. 519, 89 (1999).

[2] K. Makishima, A. Kubota, T. Mizuno, T. Ohnishi, M. Tashiro et al., Astrophys. J. 535, 632 (2000).

[3] T. Strohmayer, R. F. Mushotzky Astrophys. J. 586, L61 (2003).

[4] M. Freeland, Z. Kuncic, R. Soria, G. V. Bicknell, Mon. Not. R. Astron. Soc. 372, 630 (2006).

[5] E. Körding, E. Colbert, H. Falcke, Astron. Astrophys. 436, 427 (2005).

[6] N. A. Miller, R. F. Mushotzky, S. G. Neff, Astrophys. J. 623, L109 (2005).

[7] R. Soria, R. P. Fender, D. C. Hannikainen, A. M. Read, I. R. Stevens, Mon. Not. R. Astron. Soc. 368, 1527 (2006).

[8] R. Soria, Z. Kuncic, J. W. Broderick, S. D. Ryder, Mon. Not. R. Astron. Soc. 370, 1666 (2006).

[9] J. M. Miller, A. C. Fabian, M. C. Miller, Astrophys. J. 614, L117 (2004).

[10] A. Merloni, S. Heinz, T. Di Matteo, Mon. Not. R. Astron. Soc. 345, 1057 (2

[11] The European VLBI Network is a joint facility of European, Chinese, South African and other radio astronomy institutes funded by their national research councils.

[12] T. J. Maccarone, Mon. Not. R. Astron. Soc. 351, 1049 (2004).

[13] A. Pedlar, T. W. B. Muxlow, M. A. Garrett, P. Diamond, K. A. Wills, P. N. Wilkinson, W. Alef, Mon. Not. R. Astron. Soc. 307, 761 (1999).

[14] P. Kaaret, A. H. Prestwich, A. Zezas, S. S. Murray, D.-W. Kim, R. E. Kilgard, E. M. Schlegel, M. J. Ward, Mon. Not. R. Astron. Soc. 321, L29 (2001).

[15] A. R. McDonald, T. W. B. Muxlow, K. A. Wills, A. Pedlar, R. J. Beswick, Mon. Not. R. Astron. Soc. 334, 912 (2002).

[16] P. Kaaret, M. G. Simet, C. C. Lang, Astrophys. J. 646, 174 (2006).

[17] S. Sakai, B. F. Madore, Astrophys. J. 526, 599 (1999).

[18] A. R. King, M. B. Davies, M. J. Ward, G. Fabbiano, M. Elvis, Astrophys. J. 552, L109 (2001).

[19] S. Fabrika, A. Mescheryakov, Proc. IAU Symposium 205, p. 268 (2001).

[20] M. C. Begelman, Astrophys. J. 568, L97 (2002).

[21] A. R. King, Mon. Not. R. Astron. Soc. 335, L13 (2002).

[22] C. S. Reynolds, A. J. Loan, A. C. Fabian, K. Makishima, W. N. Brandt, T. Mizuno, Mon. Not. R. Astron. Soc. 286, 349 (1997).

[23] E. Körding, H. Falcke, S. Markoff, R. P. Fender, Astronomische Gesselschaft Abstract Series 18, P176 (2001).

[24] S. Fabrika, S. Karpov, P. Abolmasov, O. Sholukhova, Proc. IAU Symposium 230, p. 278 (2006).

[25] M. C. Begelman, A. R. King, J. E. Pringle, Mon. Not. R. Astron. Soc. 370, 399 (2006). 